BRIEF REPORT

\title{
Reduction of Acute Shoulder Dislocations in a Remote Environment: A Prospective Multicenter Observational Study
}

Therezia Bokor-Billmann, MD; Hryhoryi Lapshyn, MD; Erhard Kiffner, Prof MD; Matthias F. Goos, MD; Ulrich T. Hopt, Prof MD; Franck G. Billmann, MD, PhD

From the Department of Thoracic Surgery, University Hospital Freiburg, Freiburg, Germany (Dr Bokor-Billmann); the Department of Surgery, University Hospital Freiburg, Freiburg, Germany (Drs Lapshyn, Goos, Billmann and Prof Hopt); and the Mediface Praxisklinik, Baden Baden, Germany (Prof Kiffner).

Objective.-Acute dislocations of the glenohumeral joint are common in wilderness activities. Emergent reduction should take place at the site of trauma to reduce the patient's pain and the risk of vascular and neurological complications. A limited number of reduction methods are applicable in remote areas. The aim of this study is to present our method of reduction of anterior shoulder luxation that is easily applicable in remote areas without medication, adjuncts, and assistants and is well tolerated by patients.

Methods.-A prospective observational study was conducted during a 5-year period. The patients included underwent closed manual reduction with our technique. After each reduction, the physician who performed the reduction completed a standardized detailed history, and reexamined the patient (for acute complications). The patients were contacted 6 months after the trauma to investigate long-term postreduction complications.

Results.-Reduction was achieved with our method in 39 (100.0\%) of 39 patients. The mean pain felt during our reduction procedure was rated $1.7 \pm 1.4$ (on a scale of 10) using the visual analog scale scoring system. No complications were noted before or after the reduction attempts. We did not find any long-term complications.

Conclusions.- The reduction method presented in the present study is an effective method for the reduction of acute shoulder luxations in remote places. Our data suggest that this method could be applied for safe and effective reduction of shoulder dislocation.

Key words: shoulder dislocation, reduction, wilderness, remote place, painless

\section{Introduction}

Participation in wilderness activities has increased in the last decades. Especially in activities like alpine climbing, kayaking, or mountain biking, the enormous physical demand has led to an increase in traumatic injuries to the musculoskeletal system. ${ }^{1}$ Acute dislocations of the glenohumeral joint are common in this setting. ${ }^{2-4}$ In Europe, however, shoulder repositioning in the wilderness environment is still a controversial topic, with a lack of consensus in the medical literature. ${ }^{5}$ Considering that less than $1 \%$ of the patients in their twenties and thirties with shoulder luxation also have a fracture, ${ }^{6}$ most authors argue that emergent reduction of the dislocation should

Corresponding author: Franck Billmann MD, PhD, Department of Surgery, University Hospital Freiburg, Hugstetter Strasse 55, D-79106 Freiburg im Breisgau, Germany (e-mail: franck.billmann@ wanadoo.fr). take place at the site of trauma to reduce the patient's pain and risk of vascular and neurological complications., There are several methods of anterior dislocation reduction of the shoulder (eg, Hippocrates, Kocher, Milch, Stimson), and the success rate and complications vary according to the method used. Although most methods can be easily used in an emergency department, application in remote areas may be complicated by the lack of assistants or adjuncts (eg, repositioning chair, countertraction devices, examination table). Some techniques could be performed by the patient him or herself (eg, scapular manipulation and Stimson technique). In wilderness medicine, the ideal technique should be simple, easy to perform without assistance, quick, and atraumatic, without additional medication and relatively pain free.

The aim of this study is to present our method of reduction of anterior shoulder luxation that is easily 
applicable in remote areas without medication, adjuncts, and assistants and is well tolerated by patients. We compare our results with the previously published series (performed in a remote environment and in the emergency department) in the literature. ${ }^{8-10}$

\section{Methods}

The present study was reviewed and approved by the institutional review board at Studienberatung der Ethik Kommission, Uniklinikum Freiburg. As the present study was observational, no registration number was allocated.

\section{STUDY DESIGN}

This prospective observational study was conducted during a 5-year period from June 2009 to August 2013. Individuals participating in wilderness activities in remote areas of the Schwarzwald (Black Forest, Germany) and Vosges Mountains (France) who experienced an acute anterior shoulder dislocation and were seen by one of our volunteer rescue group physicians were included. Inclusion criteria for the study were: 1) age 16 years or older; 2) ability to give consent; 3 ) firsttime dislocations; 4) no other life-threatening injuries; and 5) repositioning maneuver performed by 1 of our 3 mountain medicine physicians. The exclusion criteria included: 1) prior sedation or analgesia; 2) dislocation obviously associated with fractures; 3 ) mental confusion; 4) refusal to consent; 5) presentation of the dislocation more than 12 hours after event; and 6) association with other traumatic injuries.

The diagnosis of shoulder dislocation was confirmed by the physician involved (typical shoulder deformation, acute pain, acute impairment of the range of motion of the shoulder joint). The combination of these signs was considered sufficient to exclude subluxations or spontaneous reductions. All the patients included underwent closed manual reduction with our technique. In these remote places, no prereduction x-ray was performed. The diagnosis of shoulder dislocation was solely based on clinical examination. Successful reduction was determined by clinical signs and symptoms, including absence of pain, full recovery of range of motion, and normal appearance of the shoulder. Neurovascular examinations were performed before and after reduction.

After each reduction, the physician who performed the reduction completed a standardized detailed history including demographic data and a brief medical history. The mechanism of injury, the time interval between injury and first attempt at reduction, the duration of the reduction (measured using a timer), the number of attempts, and the existence of complications before and after reduction were registered. The reduction was characterized as unsuccessful after a failed second attempt. After successful reduction, each patient was asked to rate the degree of pain he or she felt during reduction using a visual analog scale (VAS: 0 points representing no pain, 10 points representing intolerable pain).

Each patient was asked to undergo an x-ray (anteroposterior and axillary views) of the shoulder within 2 weeks after reduction (once they had returned to the front country) to rule out a shoulder injury (eg, fractures, acromioclavicular separation). These x-ray investigations were not performed at the same institution. The patients were contacted 6 months after the trauma to investigate long-term postreduction complications (eg, neurovascular complications, shoulder instability), the result of the $\mathrm{x}$-ray investigation, and the need for delayed surgery.

\section{REDUCTION TECHNIQUE}

The reduction technique used in the present study requires only 1 practitioner (see Video and Figure 1). No specific equipment is necessary. This technique was new for the 3 physicians involved. The present reduction technique was adapted from the technique taught during the mountain medicine course at the mountain medicine university diploma (Université Paris 13, France). Anatomically, the 5 steps move the head of the humerus into a position where the greater tuberosity of the humerus neck is the smallest and has therefore a maximal chance to slide over the scapular border. The patient is seated in a straight-back position against a firm surface, with a rucksack or snow wall to minimize movements of the upper body. All manipulations are performed very slowly to prevent pain and to guarantee optimal muscle relaxation. Throughout the reduction maneuver, there is no need for traction (or countertraction) or forced movements; the patient must feel as relaxed and comfortable as possible. The application of our technique involves the following steps:

1. The practitioner holds the patient's wrist with the left hand (in the case of a left shoulder dislocation) and the patient's elbow with the right hand.

2. With the elbow in $90^{\circ}$ of flexion, the glenohumeral joint is flexed forward to $90^{\circ}$.

3. While still in flexion, the glenohumeral joint is adducted until the elbow reaches the midline of the body; it is important to continue this movement until this landmark is completely reached.

4. Then, internal rotation of the shoulder is performed. During this step, the patient's elbow must stay at the landmark described above. At $25^{\circ}$ to $30^{\circ}$ of rotation, a mild resistance is usually encountered. 


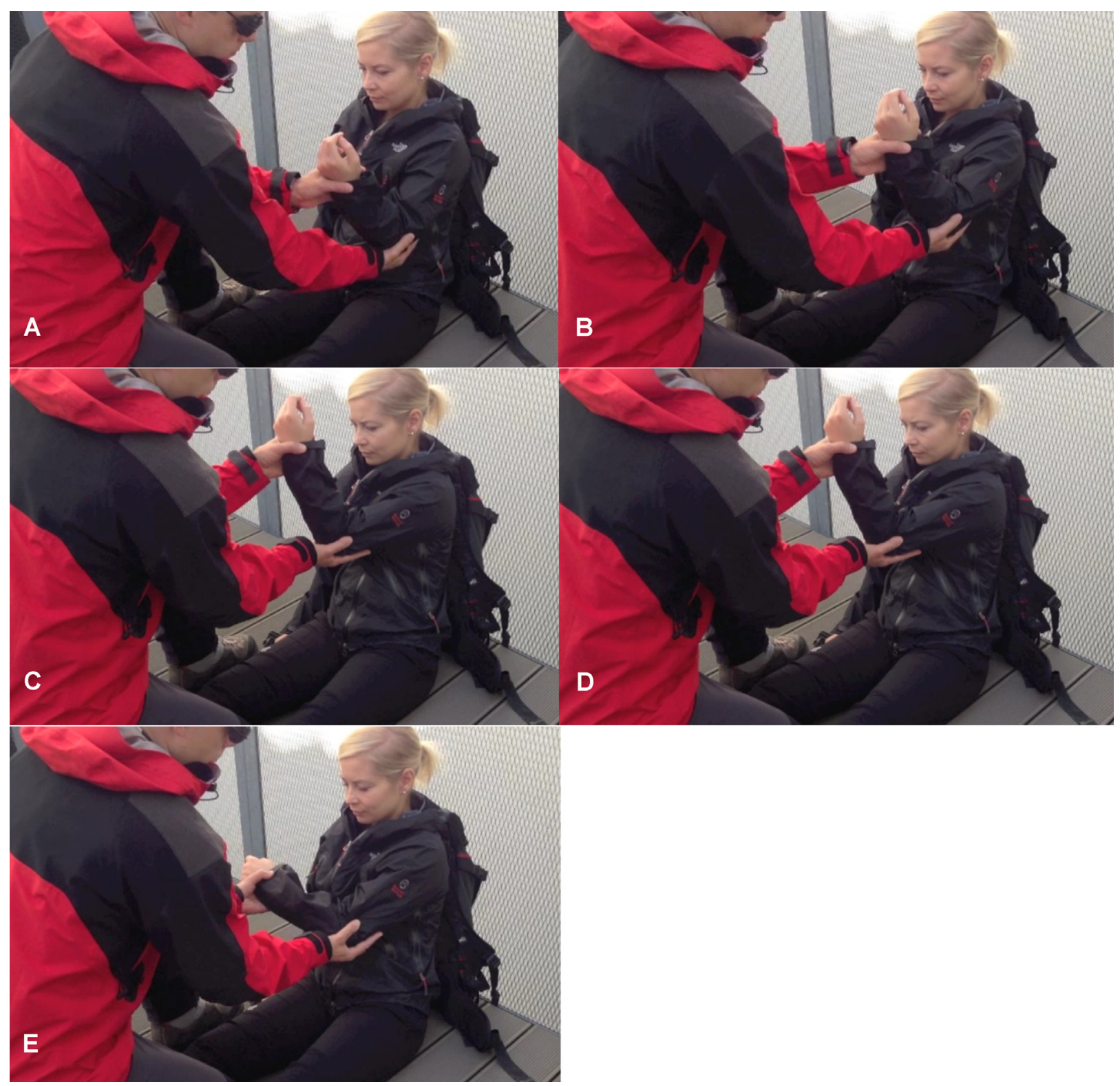

Figure 1. Diagram depicting the steps of our reduction procedure. (A) The practitioner holds the patient's wrist with the left hand (in the case of a left shoulder dislocation) and the patient's elbow with the right hand. (B) With the elbow in $90^{\circ}$ of flexion, the glenohumeral joint is flexed forward to $90^{\circ}$. (C) While still in flexion, the glenohumeral joint is adducted until the elbow reaches the midline of the body; it is important to continue this movement until this landmark is completely reached. (D) Then, internal rotation of the shoulder is performed. During this step, the patient's elbow must stay at the landmark described above. At $25^{\circ}$ to $30^{\circ}$ of rotation, a mild resistance is usually encountered. (E) The last step of the maneuver consists of applying a constant internal rotation pressure to overcome this mild resistance without pain. Reduction is usually achieved at approximately $30^{\circ}$ of internal rotation.

5. The last step of the maneuver consists of applying a constant internal rotation pressure to overcome this mild resistance without pain. Reduction is usually achieved at approximately $30^{\circ}$ of internal rotation.

All reductions reported here were performed without sedation, anesthesia, or pain medication. Although
5 physicians were involved in the present study, only 3 of them performed reduction in the field. The other 2 worked on the elaboration of the study design and the preparation of the manuscript. All 5 physicians involved in this study attended a brief instructional course (1 hour theoretical and 2 hours practical course depicting the anatomical basis and the key points of the technique) before involvement in the study. 


\section{DATA ANALYSIS AND STATISTICS}

Descriptive statistics were calculated using STATA/IC version 13.1 (Stata Corp, College Station, TX).

\section{Results}

Demographics and mechanism of injury are summarized in Table 1. The reduction result and the time needed for reduction and from injury to reduction, as well as the pain felt during the reduction and the complications, are summarized in Table 2.

\section{DEMOGRAPHICS}

Thirty-nine patients ( 31 men, 8 women) were included in the present prospective observational study. The mean age $( \pm \mathrm{SD})$ was $42.3 \pm 16.3$ years. The demographic characteristics of the patients studied are shown in Table 1.

\section{MECHANISM OF INJURY}

The majority of patients made the diagnosis of shoulder luxation on their own. No luxation was clinically accompanied by signs of humeral or scapular fracture. This was confirmed in the postreduction x-ray examinations. The mechanisms of dislocation are shown in Table 1.

\section{REDUCTION RESULT AND TIME}

Reduction was achieved with our method in 39 (100.0\%) of 39 patients. Reduction results, reduction time, and the VAS pain score before, during, and after reduction are summarized in Table 2. Reduction duration was relatively long with our method $(6.1 \pm 1.4$ minutes $)$ in comparison with other techniques.

Table 1. Demographics and mechanism of injury encountered by patients

\begin{tabular}{lc}
\hline Variable & Value \\
\hline Age (years), mean $\pm \mathrm{SD}$ & $42.3 \pm 16.3$ \\
Sex & \\
$\quad$ Men, n (\%) & $31(79.5)$ \\
$\quad$ Women, n (\%) & $8(20.5)$ \\
Hand dominance left/right, n & $7 / 32$ \\
Side of dislocation left/right, n & $17 / 22$ \\
Mechanism of dislocation & \\
$\quad$ Alpine climbing, n (\%) & $12(30.8)$ \\
Skiing, n (\%) & $5(12.8)$ \\
Kayaking, n (\%) & $9(23.1)$ \\
Other, n (\%) & $13(33.3)$ \\
\hline
\end{tabular}

Table 2. Efficacy, time needed for reduction, pain felt during the reduction, and complications in the present series

\begin{tabular}{lc}
\hline Variable & Value \\
\hline Reduction result, n (\%) & \\
$\quad$ Success at first attempt & $37(94.9)$ \\
Success at second attempt & $2(5.1)$ \\
Failure & $0(0.0)$ \\
Time interval (hours), mean $\pm \mathrm{SD}^{a}$ & $3.9 \pm 2.1$ \\
Reduction time (min), mean $\pm \mathrm{SD}^{b}$ & $6.1 \pm 1.4$ \\
VAS pain score, mean $\pm \mathrm{SD}$ & $1.7 \pm 1.4$ \\
Complications & $0(0.0)$ \\
$\quad$ Preprocedure, n (\%) & $0(0.0)$ \\
Postprocedure, n (\%) & $0(0.0)$ \\
Need for surgery after reduction & \\
\hline
\end{tabular}

VAS, visual analog scale.

${ }^{a}$ Between the dislocation and the first attempt at reduction.

${ }^{b}$ Between the attempt beginning and the successful reduction.

\section{PAIN DURING REDUCTION}

The mean pain felt during our reduction procedure was rated $1.7 \pm 1.4$ using the VAS scoring system.

\section{TIME FROM INJURY TO REDUCTION}

The mean time interval $( \pm \mathrm{SD}$ ) from injury to first reduction attempt was $3.9 \pm 2.1$ hours.

\section{COMPLICATIONS}

No complications were noted before or after the reduction attempts in any of the studied patients. We found neither long-term complications nor need for surgery in the months after reduction.

\section{Discussion}

Patients experiencing shoulder dislocation were mostly young men $(79.5 \%)$ who participated in activities like alpine climbing, skiing, or kayaking, although other mechanisms of injury were seen (eg, trail running, mountain biking). These results are consistent with prior series in the literature. ${ }^{11-13}$

Several studies have evaluated the efficacy of different methods of reduction of anterior dislocation of the shoulder. Methods like the Kocher method without traction, the arm hang or Stimson, or scapular manipulation use limited force and can be performed without sedation. According to the literature, the success rates of these methods (with and without sedation) ranged between $60.0 \%$ and $100.0 \%$ (Table 3). ${ }^{8-10}$ The on-scene success rate of $100.0 \%$ for our reduction method (first and second attempts) is comparable with the highest success rates for 
Table 3. Reduction techniques and their success rates in the literature

\begin{tabular}{lc}
\hline Reduction technique & Success rate $(\%)^{a}$ \\
\hline Arlt & 97 \\
Chair & 72 \\
External rotation & 90 \\
Kocher & 82 \\
Milch & 95 \\
Scapular manipulation & 100 \\
Spaso & 87 \\
Self reduction & 60 \\
Walz & 90 \\
\hline
\end{tabular}

${ }^{a}$ The best value was retained.

single attempts in the emergency department (70\% to $96 \%$ depending on the method used). ${ }^{8-10,14}$ This result is notable for the fact that the reduction is performed without sedation and in a remote environment. Moreover, the technique itself is easy to apply (see Video at http://dx.doi.org/10.1016/ j.wem.2014.12.027) and can be performed without need of traction, countertraction, and other external aids. The slowness of our technique is mandatory for a low pain level during reduction. Moreover, the fact that our method was performed without sedation and with only mild pain counterbalances the time needed for reduction. In the 2 cases in which the first reduction attempt failed, the duration of this first attempt was up to 10 minutes (9.5 and 10 minutes). Thus, a rushed technique could not be the explanation for the failed attempt. We hypothesize that patients were not relaxed sufficiently for the reduction to be successful.

The pain that was felt during the reduction was mild with our method (VAS, $1.7 \pm 1.4$ ) in comparison with other series. ${ }^{11-13}$ This is remarkable considering that our reduction method was carried out without sedation. However, this result is comparable to other low-energy techniques that have been used in a prehospital and hospital setting. There is also a trend in US emergency departments to reduce shoulders without sedation, using specific techniques (eg, scapular manipulation, arm hangs, or Cunningham technique) or adjuncts such as intra-articular blocks. ${ }^{14}$ We propose that our method is also suitable for a remote environment.

The mean time to reduction was 3.9 hours for the present method. The prior series investigating time from injury to reduction reported rapid dislocation to reduction intervals. That can be explained by the fact that these investigations did not take place in remote environments. Compared with the results of other series, ${ }^{8,12}$ our results (3.9 hours) in remote environments highlight the advantage of on-site treatment. Early reduction has been shown to be beneficial in maintaining neurovascular integrity of the affected patient and to reduce early and late complications. ${ }^{15}$

No acute or long-term complications after on-scene reduction were diagnosed in the patients included in the present study. This result is consistent with other studies investigating reduction performed in medical or wilderness environments. ${ }^{12,13}$ However, the long-term survey did not include a clinical examination.

Our study has several limitations. The present investigation is an observational study; thus, it lacks power as a result of the method used. Further, we did not perform an examination of ligamentous laxity that could have influenced the ease and speed of reduction, nor did we perform a clinical examination to diagnose long-term complications after reduction. Reductions were performed by physicians; thus, we cannot generalize our results to nonphysician practitioners. Diagnosis of dislocation was not confirmed by x-ray, which can be considered as a further limitation. We did not directly compare our reduction technique with other techniques. Finally, we included a limited number of patients; this can affect the statistical relevance of our results.

In conclusion, the reduction method presented in the present study is an effective method for the reduction of acute shoulder luxations in remote places. Moreover, we hypothesize that some of the traditional techniques would be more successful if performed slowly and without excessive force, and that our method could be applied by lay providers for safe and effective reduction of shoulder dislocations.

\section{Acknowledgments}

Our gratitude goes to Prof Jean-Paul Richalet (Chef du Service de Médecine du Sport, Hôpital Avicenne; Directeur du Laboratoire de Réponses Cellulaires et Fonctionnelles à l'Hypoxie, Bobigny, France) and Dr Paul Robach (École Nationale de Ski et d'Alpinisme, Chamonix, France) for their mountain medicine education and their advice.

We want particularly to thank Dr Joanne Eysell for her help in checking the English language.

\section{Supplementary data}

Supplementary data associated with this article can be found in the online version at http://dx.doi.org/10.1016/j. wem.2014.12.027.

\section{References}

1. Marsigny B, Lecoq-Jammes F, Cauchy E. Medical mountain rescue in the Mont-Blanc massif. Wilderness Environ Med. 1999;10:152-156. 
2. Fiore DC. Injuries associated with whitewater rafting and kayaking. Wilderness Environ Med. 2003;14:255-260.

3. Flores AH, Haileyesus T, Greenspan AI. National estimates of outdoor recreational injuries treated in emergency departments, United States, 2004-2005. Wilderness Environ Med. 2008;19:91-98.

4. Kocher MS, Feagin JA Jr. Shoulder injuries during alpine skiing. Am J Sports Med. 1996;24:665-669.

5. Flinn SD. On-field management of emergent and urgent extremity conditions. Curr Sports Med Rep. 2006;5:227-232.

6. Orloski J, Eskin B, Allegra PC, Allegra JR. Do all patients with shoulder dislocations need prereduction x-rays? Am J Emerg Med. 2011;29:609-612.

7. Pasila M, Jaroma H, Kiviluoto O, Sundholm A. Early complications of primary shoulder dislocations. Acta Orthop Scand. 1978;49:260-263.

8. Ditty J, Chisholm D, Davis SM, Estelle-Schmidt M. Safety and efficacy of attempts to reduce shoulder dislocations by non-medical personnel in the wilderness setting. Wilderness Environ Med. 2010;21:357-361.e2.

9. Ufberg JW, Vilke GM, Chan TC, Harrigan RA. Anterior shoulder dislocations: beyond traction-countertraction. J Emerg Med. 2004;27:301-306.
10. Walz M, Kolbow B, Auerbach F. A simple, gentle and painless technique for reduction of anterior shoulder dislocation [in German]. Unfallchirurg. 2006;109:551555.

11. Amar E, Maman E, Khashan M, Kauffman E, Rath E, Chechik O. Milch versus Stimson technique for nonsedated reduction of anterior shoulder dislocation: a prospective randomized trial and analysis of factors affecting success. J Shoulder Elbow Surg. 2012;21:1443-1449.

12. Sayegh FE, Kenanidis EI, Papavasiliou KA, Potoupnis ME, Kirkos JM, Kapetanos GA. Reduction of acute anterior dislocations: a prospective randomized study comparing a new technique with the Hippocratic and Kocher methods. J Bone Joint Surg Am. 2009;91:2775-2782.

13. Singh S, Yong CK, Mariapan S. Closed reduction techniques in acute anterior shoulder dislocation: modified Milch technique compared with traction-countertraction technique. J Shoulder Elbow Surg. 2012;21:1706-1711.

14. Cunningham NJ. Techniques for reduction of anteroinferior shoulder dislocation. Emerg Med Australas. 2005;17: 463-471.

15. Iserson KV. Relocating dislocations in a wilderness setting: use of hypnosis. J Wilderness Med. 1991;2:22-26. 